\title{
Transsinusoidal Portal Vein Embolization with Ethylene Vinyl Alcohol Copolymer (Onyx): A Feasibility Study in Pigs
}

\author{
Maarten L. J. Smits • Peter Vanlangenhove $\cdot$ Emiel J. C. Sturm • \\ Maurice A. A. J. van den Bosch • Monirath Hav • Marleen Praet • \\ Maarten A. D. Vente $\cdot$ Frédéric R. Snaps $\cdot$ Luc Defreyne
}

Received: 25 July 2011/Accepted: 18 September 2011/Published online: 7 October 2011

(C) The Author(s) 2011. This article is published with open access at Springerlink.com

\begin{abstract}
Purpose Portal vein embolization is performed to increase the future liver remnant before liver surgery in patients with liver malignancies. This study assesses the feasibility of a transsinusoidal approach for portal vein embolization (PVE) with the ethylene vinyl alcohol copolymer, Onyx.

Methods Indirect portography through contrast injection in the cranial mesenteric artery was performed in eight healthy pigs. Onyx was slowly injected through a microcatheter from a wedged position in the hepatic vein and advanced through the liver lobules into the portal system. The progression of Onyx was followed under fluoroscopy, and the extent of embolization was monitored by indirect portography. The pigs were euthanized immediately $(n=2)$, at 7 days $(n=4)$, or at 21 days postprocedure $(n=2)$. All pigs underwent necropsy and the ex vivo livers were grossly and histopathologically analyzed.
\end{abstract}

M. L. J. Smits - M. A. A. J. van den Bosch - M. A. D. Vente Department of Radiology, University Medical Center Utrecht, Heidelberglaan 100, HP E01.13, 3584 XC Utrecht,

The Netherlands

e-mail: m.1.j.smits-3@umcutrecht.nl

M. A. A. J. van den Bosch

e-mail: mbosch@umcutrecht.nl

M. A. D. Vente

e-mail: m.vente@umcutrecht.nl

P. Vanlangenhove · E. J. C. Sturm · L. Defreyne $(\square)$

Department of Vascular and Interventional Radiology, Ghent

University Hospital, De Pintelaan 185, 9000 Ghent, Belgium

e-mail: luc.defreyne@ugent.be

P. Vanlangenhove

e-mail: peter.vanlangenhove@uzgent.be
Results Transsinusoidal PVE was successfully performed in five of eight pigs (63\%). In 14 of 21 injections (67\%), a segmental portal vein could be filled completely. A mean of 1.6 liver lobes per pig was embolized (range 1-2 lobes). There were no periprocedural adverse events. Focal capsular scarring was visible on the surface of two resected livers, yet the capsules remained intact. Histopathological examination showed no signs of recanalization or abscess formation. Mild inflammatory reaction to Onyx was observed in the perivascular parenchyma.

Conclusions The porcine portal vein can be embolized through injection of Onyx from a wedged position in the hepatic vein. Possible complications of transsinusoidal PVE and the effect on contralateral hypertrophy need further study.

Keywords Liver - Portal vein embolization - Wedged hepatic venography $\cdot$ Pig · Onyx

\author{
E. J. C. Sturm \\ e-mail: ejcsturm@gmail.com \\ M. Hav - M. Praet \\ N. Goormaghtigh Institute of Pathology, Ghent University \\ Hospital, De Pintelaan 185, 9000 Ghent, Belgium \\ e-mail: hav.monirath@gmail.com \\ M. Praet \\ e-mail: marleen.praet@ugent.be \\ F. R. Snaps \\ Department of Clinical Sciences, Faculty of Veterinary \\ Medicine, University of Liège, Boulevard de Colonster B41, \\ 4000 Liège, Belgium \\ e-mail: fsnaps@ulg.ac.be
}




\section{Introduction}

Patients with primary or secondary liver tumors have the highest chance of cure when treated by surgical resection. In many of these patients, however, the future liver remnant (FLR), i.e., the part of the liver that remains in situ after resection, is insufficient to compensate the loss of liver capacity $[1,2]$. To increase the volume of the FLR and render the patient an adequate candidate for surgery, preoperative portal vein embolization (PVE) can be performed [3-5]. In PVE, the branches of the portal vein that supply the affected liver segments (generally the right lobe) are embolized 2-3 weeks before resection. As a result, hypertrophy of the nonembolized segments (generally the left lobe) is induced.

Approaching the portal vein for embolization constitutes a technical challenge. The portal vein is not directly connected to the central venous system and therefore cannot be entered via the usual access sites for venous interventions (e.g., femoral vein, jugular vein, or subclavian vein). Access to the portal vein is classically gained by a percutaneous transhepatic puncture but also can be achieved via catheterization of the ileocolic vein at laparotomy [6, 7]. A complication rate of $12.8 \%$ has been reported for transhepatically performed PVE, comprising subcapsular hematoma, hemoperitoneum, and migration of emboli or coils into a portal vein branch supplying the FLR [8]. Although this is an acceptable complication rate, many of these complications can be attributed solely to the transhepatic approach. The ideal approach to perform PVE would be nonoperative, not requiring a transhepatic needle track, not disturbing the integrity of the liver vessels, nor jeopardizing the FLR.

In search for alternative approaches, Perarnau et al. successfully performed transjugular PVE in humans, based on the method of creating a transjugular intrahepatic portosystemic shunt (TIPS) [9]. Madoff et al. described a method to embolize the portal vein transarterially [10]. This method was investigated in five pigs and proved to be a safe and effective way to induce FLR hypertrophy.

We present another technique for PVE inspired by the observation that at wedged hepatic venography, contrast material can retrogradely traverse the sinusoids and fill the portal vein [11-13]. We hypothesized that PVE could equally be performed by retrograde injection of a liquid embolic agent from a wedged catheter in the hepatic vein. In this study, we investigated the feasibility of this approach by performing this procedure in a swine model using the low-viscosity liquid embolic agent, Onyx.

\section{Materials and Methods}

\section{Study Design and Subjects}

The feasibility of transsinusoidal PVE was investigated in eight healthy female pigs of the Piétrain race (body weight $25-32 \mathrm{~kg}$ ). The objectives were to embolize the left hemiliver in a limited number of pigs $(n=2)$ and to embolize the right hemiliver in the rest of the pigs $(n=6)$. The right hemiliver was more frequently targeted, because this reflects the predominant clinical need for PVE of the right hemiliver. This study was performed in agreement with the institutional and national Guide for the Care and Use of Laboratory Animals, and the European Convention for the Protection of Vertebrate Animals used for Experimental and Other Scientific Purposes (1986), and approved by the institutional animal care and use committee under ethical agreement number 693 .

Apart from a rudimentary caudate lobe, four liver lobes can be differentiated in the porcine liver: the right lateral lobe, the right medial lobe, the left medial lobe, and the left lateral lobe (Fig. 1A). In contrast to the bifurcated human portal vein, the porcine portal vein can be described as a curved trunk out of which four lobar branches emerge [14, 15]. The lobar branches divide into segmental branches for each individual segment of that lobe. We will refer to these branches as segmental portal veins. Our purpose was not to occlude all the small side branches of the portal system but to push Onyx up to the portal trunk and occlude at least one segmental portal vein per injection. Each injection of Onyx was only considered successful if at least one segmental portal vein could be embolized up to its origin. The overall procedure was considered successful if the portal veins of the entire targeted hemiliver were embolized completely.

\section{Embolic Agent}

Ethylene vinyl alcohol copolymer (Onyx, ev3, Irvine, CA, USA) was used for the experiments. Onyx is a nonadhesive liquid embolic agent that consists of ethylene vinyl alcohol $(\mathrm{EVOH})$ dissolved in dimethyl sulfoxide (DMSO). Micronized tantalum powder is added to the suspension to provide radiopacity for visualization under fluoroscopy. Inside the vessel, DMSO dissipates into the blood and interstitium and Onyx forms a spongy, coherent embolus that does not adhere to the vessel wall [16]. Onyx is available in different solutions: Onyx-18 (6\% EVOH), Onyx-20 (6.5\% EVOH), Onyx-34 (8\% EVOH), and Onyx$500(20 \% \mathrm{EVOH})$. The concentration of EVOH is directly related to the viscosity of the injectable substance. For these experiments, Onyx-18 was used to obtain deeper penetration of the vascular structure, as recommended by 

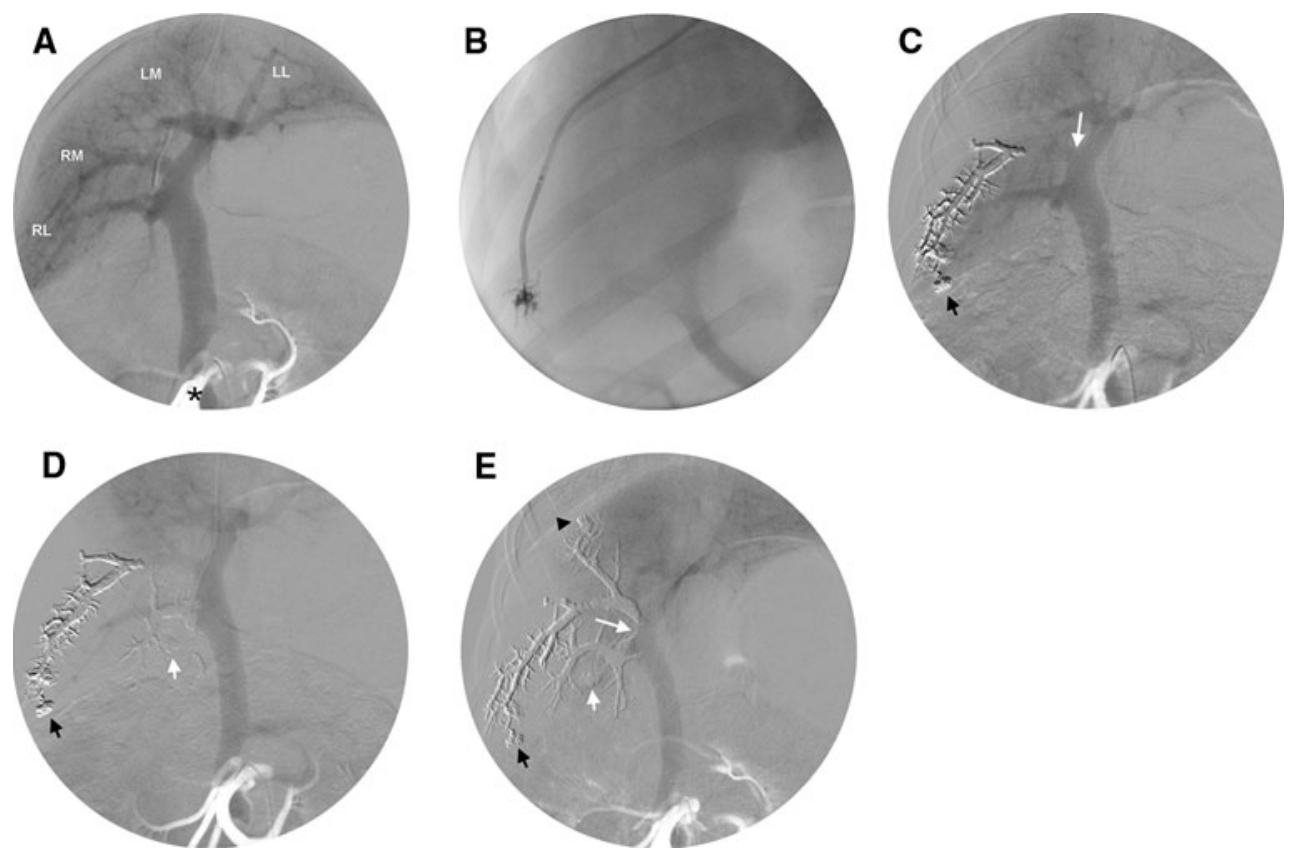

Fig. 1 A Preembolization portogram showing the portal supply to the right lateral lobe $(R L)$, the right medial lobe $(R M)$, the left medial lobe $(L M)$, and the left lateral lobe $(L L)$, and the position of the arterial catheter in the cranial mesenteric artery (*). B Injection of Onyx from a wedged catheter position in the hepatic vein. $\mathbf{C}$ Onyx cast is visible at portography after first injection (injection position marked by the black short arrow). This injection was unsuccessful, because the segmental portal vein was not embolized up to its origin (white long arrow). D Onyx cast during the second injection (injection position

the manufacturer [17]. In addition, Komemushi et al. [18] have demonstrated that the penetration of Onyx through peripheral blood vessels is best for the lowest concentrations of EVOH.

\section{Angiographic Procedure}

The pigs were placed in supine position and orotracheally intubated after sedation with intramuscular injection of ketamine $(20 \mathrm{mg} / \mathrm{kg})$ plus midazolam $(0.1 \mathrm{mg} / \mathrm{kg})$. Anesthesia was obtained and maintained with isoflurane gas (2\%) plus oxygen $(1 \mathrm{l} / \mathrm{min})$. Intravascular access to the common femoral artery and the internal jugular vein and/or femoral vein was obtained via the Seldinger technique [19]. 6-French (F) access sheaths (Radiofocus introducer II, Terumo Europe, Leuven, Belgium) were introduced at the vascular access sites. The cranial mesenteric artery was catheterized with a 5-F diagnostic Cobra catheter (Cook, Bjaeverskov, Denmark) over a diagnostic guidewire (Fixed Core Wire Guide 0.035 "; Cook) and $20 \mathrm{ml}$ of radiopaque contrast (iodixanol, Visipaque $320 \mathrm{mg} \mathrm{I} / \mathrm{ml}$, GE Healthcare, Diegem, Belgium) was administered to obtain an indirect portogram using a digital C-arm angiography system (Siremobil; Siemens Medical Solutions, Erlangen, marked by the white short arrow) successfully embolizing the RL portal vein. E Final portography shows a successfully embolized right hemiliver after the RM portal vein had been embolized by the third injection (injection position marked by the black arrowhead). Note that the Onyx cast from the second injection extends into the portal trunk (white long arrow). The diameter of the portal vein has markedly decreased compared with the preembolization portogram (A)

Germany). A 5-F catheter was introduced through the jugular or femoral venous sheath and advanced into the hepatic vein where it was placed in wedge. Then, a microcatheter (Rebar, DMSO-compatible, 0.010" or 0.014", ev3, Irvine, CA, USA) was advanced over a hydrophilic microguidewire (Silverspeed 0.010", ev3, Irvine, CA, USA) until this microcatheter reached a wedged position in the hepatic vein as well. This position was approximately 5-10 $\mathrm{mm}$ distal to the tip of the guiding catheter.

\section{Embolization}

When a wedged position of the microcatheter was obtained, wedged hepatic venography was performed by a forceful hand injection of contrast medium. In case the contrast medium clearly filled the lumen of a target segmental portal vein at wedged hepatic venography, the microcatheter was primed with DMSO (ev3) with a volume equal to the death space of the microcatheter to prevent preterm solidification of Onyx. Onyx was then slowly injected at approximately $0.1 \mathrm{ml} / \mathrm{min}$ under subtraction fluoroscopy while observing its retrograde progression through the sinusoids into the portal system (Fig. 1B). Injection rate and injected amount of Onyx were registered. 
During injection, particular attention was paid to possible reflux of Onyx toward the guiding catheter. Two types of reflux had to be recognized: reflux along the microcatheter toward the guiding catheter [20], and reflux into a communicating branch of the catheterized hepatic vein (venovenous communications) through which Onyx could escape antegradely instead of progressing retrogradely through the sinusoids of the liver lobule [13]. In case of reflux, the injection was paused for 1 minute to allow solidification of intravascular Onyx. If reflux persisted or if the segmental portal vein could not be fully embolized, the injection was deemed unsuccessful and another central vein was catheterized. The procedure was stopped when the aimed part of the portal system was embolized or when the embolic agent advanced to untargeted portal segments. The extent of embolization was examined during and after the procedure by indirect portography. The pigs were sacrificed by an overdose of pentobarbital immediately $(n=2), 7$ days $(n=4)$, or 21 days after the procedure $(n=2)$. The pigs that were not immediately sacrificed received a single dose of intramuscular antibiotics (amoxicillin, $30 \mathrm{mg} / \mathrm{kg}$ ). All pigs underwent necropsy during which the livers and lungs were collected. The ex vivo lungs were inspected under fluoroscopy and were evaluated at gross examination and histopathology to rule out Onyx emboli. The livers were macroscopically viewed for any visible defects (e.g., capsular penetration, ulceration, or hematoma), and fluoroscopy images were obtained from all ex vivo livers to match the extent of Onyx with the angiograms obtained during the procedure.

\section{Number of Involved Lobules}

To improve our understanding of the mechanism of transsinusoidal PVE, we theoretically reconstructed the pathway of the Onyx from the microcatheter to the portal venule. To know how many lobules are involved in a typical injection with Onyx, we estimated the number of central veins that supply the wedged hepatic vein by applying the fractal law for vessel branching [21]. Presuming that the vascular branching follows an area-preserving pattern like most vascular structures, the crosssectional surface of a parent vessel equals the sum of that of the daughter vessels. By applying the fractal law formula $\pi r_{k}^{2}=n \pi r_{k+1}^{2}$, in which $r_{k}$ is the radius of the hepatic vein at the level of the wedged position and $r_{k+1}$ is the radius of the central vein of a hepatic lobule, we calculated the number of central veins $(n)$ that were theoretically involved in the transsinusoidal PVE. We assumed that the radius of the wedged hepatic vein $\left(r_{k}\right)$ was equal to the outer diameter of the microcatheter divided by two (because the microcatheter was in wedged position). For the radius of the central vein $\left(r_{k+1}\right)$, we measured the mean radius of 50 central veins of two untreated pigs using a digital microscope (Mirax midi, Carl Zeiss Microimaging, Jena, Germany). These two pigs were of the same race and similar body weight as the pigs that underwent transsinusoidal PVE. Each lobule has one central vein; therefore, the number of involved central veins and the number of involved lobules are interchangeable.

\section{Histopathological Evaluation}

Specimens were obtained from multiple parts of the embolized and nonembolized liver segments. The specimens were sectioned at 4 microns, stained with hematoxylin and eosin (H\&E) and Masson's trichrome and were analyzed under light microscope at $\times 40$ magnification. First, the above-described formula to calculate the number of involved lobules was compared with the actual number of Onyx-filled central veins surrounding the injection site on the histopathological specimens. Second, the embolized and nonembolized segments were evaluated for the presence and location of Onyx, signs of fibrosis, necrosis, abscess formation, perivascular tissue reaction, and vascular recanalization.

\section{Results}

The portal veins supplying the target hemilivers were successfully embolized in five of eight pigs (63\%; Fig. 1). In three of eight pigs (37\%), one of the two target liver lobes could not be embolized. In these cases, we were unable to find an appropriate wedged position in the draining hepatic vein of these liver lobes. A total of 21 injections were performed, 14 of which were successful (67\%; Table 1). During these successful injections, Onyx could be smoothly pushed retrogradely through the lobules until a segmental portal vein was embolized up to its origin. The unsuccessful injections were characterized by swelling of the injection site (3/7) or by early reflux that could not be solved by 1 min of waiting (4/7). Reflux of Onyx occurred in four of 14 successful cases (29\%); in total, eight of 21 injections (38\%) were complicated by reflux. In two of 14 successful cases (14\%), Onyx antegradely flowed beyond the tip of the microcatheter. This type of reflux was resolved in both cases after $1 \mathrm{~min}$ of waiting. In another two cases (14\%), Onyx escaped antegradely through veno-venous communications. Overall, a mean volume of $6.8 \mathrm{ml}$ of Onyx was injected per pig (range 3.3-9.3 ml). Successful injections required a mean volume of $3.0 \mathrm{ml}$ of Onyx. There were seven unsuccessful injections (33\%), during which Onyx could not be advanced through the lobules. Accumulation of Onyx was then observed around the injection site. In three of seven 
Table 1 Experiment details

$R L$ right lateral liver lobe; $R M$ right medial liver lobe; $L L$ left lateral liver lobe; $L M$ left medial liver lobe

\begin{tabular}{ll}
\hline & $n$ \\
\hline Study subjects & \\
No. of pigs & 8 \\
Targeted liver lobes & 6 \\
RL + RM & 2 \\
LL + LM & $5 / 8(63 \%)$ \\
No. of pigs successfully embolized & $21(2.6)$ \\
Total no. of injections (mean per pig) & $14 / 21(67 \%)$ \\
No. of successful injections & $7 / 21(33 \%)$ \\
No. of unsuccessful injections & $6.8(3.3-9.0)$ \\
Amount of Onyx injected per pig (ml), & \\
mean (range) & $234(144-206)$ \\
Duration of procedure (min), & \\
mean (range) & \\
Time between Onyx injections & \\
and euthanasia & \\
0 days & $2 / 8(25 \%)$ \\
7 days & $4 / 8(50 \%)$ \\
21 days & $2 / 8(25 \%)$ \\
\hline
\end{tabular}

unsuccessful injections, another site was found to wedge the catheter system and successfully inject Onyx. In one case, the Onyx embolus extended into the portal trunk after a segmental portal vein had been overfilled (Fig. 1E).

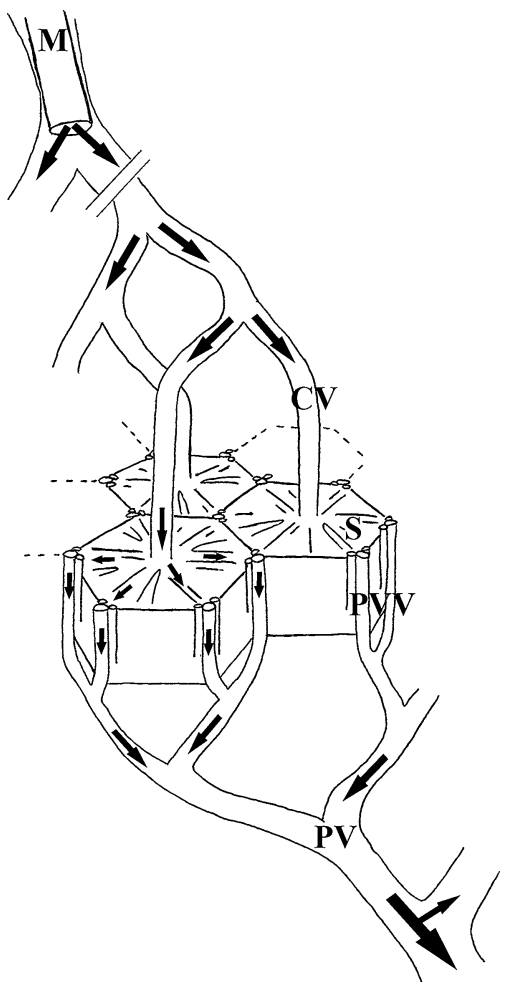

Fig. 2 Pathway of Onyx (arrows) from the wedged microcatheter $(M)$ in the hepatic vein trough the central veins $(C V)$ and the sinusoids $(S)$ of the hexagonal shaped liver lobules, into the portal venules $(P V V)$ and branches of the portal vein $(P V)$
Fortunately, this caused no flow obstruction in the portal trunk. All branches to untargeted segments stayed patent and free of Onyx on portographic and histopathologic control. Marked vasoconstriction of the portal vein was observed in two of eight pigs after the procedure.

Figure 2 provides a schematic view of the pathway of Onyx injected from the wedged position through the lobules into the portal system. The microcatheters that we used had outer diameters of $0.57-0.60 \mathrm{~mm}$, so $r_{k}$ (radius of the wedged hepatic vein) equals $0.29-0.30 \mathrm{~mm}$ respectively. Digital microscope measurement of the radius of the central vein in the two untreated animals returned a mean $r_{k+1}$ (radius of the central vein) of $0.06 \mathrm{~mm} \pm 0.01$. By applying the formula: $\pi r_{k}^{2}=n \pi r_{k+1}^{2}$, the number of central veins $(n)$ through which the Onyx passes would theoretically equal 23 .

After the procedure, the six pigs that were kept alive did not show any signs of distress and they resumed their usual activity pattern within a few hours. Necropsy did not reveal any anomalies. No traces of Onyx were found in the lungs under fluoroscopy or at histopathological evaluation. Gross examination of the ex vivo livers of two pigs exhibited surface irregularities, which were consistent with superficially embolized sinusoids causing swelling and scarring of perivascular tissue (Fig. 3). The liver capsule was, however, intact. Radiographic images of the resected livers showed no displacement of Onyx compared with the angiograms of the procedure. The liver of one of the pigs (sacrificed 7 days postprocedurally) was unfortunately excluded from histopathological assessment due to insufficient fixation with $4 \%$ formaldehyde. 
Fig. 3 A Macroscopic view of a capsular lesion (arrow), consistent with $\mathbf{B}$ a superficial injection site (arrow) under fluoroscopy. C Microscopic view (H\&E, original magnification $\times 40$ ) of the same region shows capsular fibrosis (arrow) around the injection site
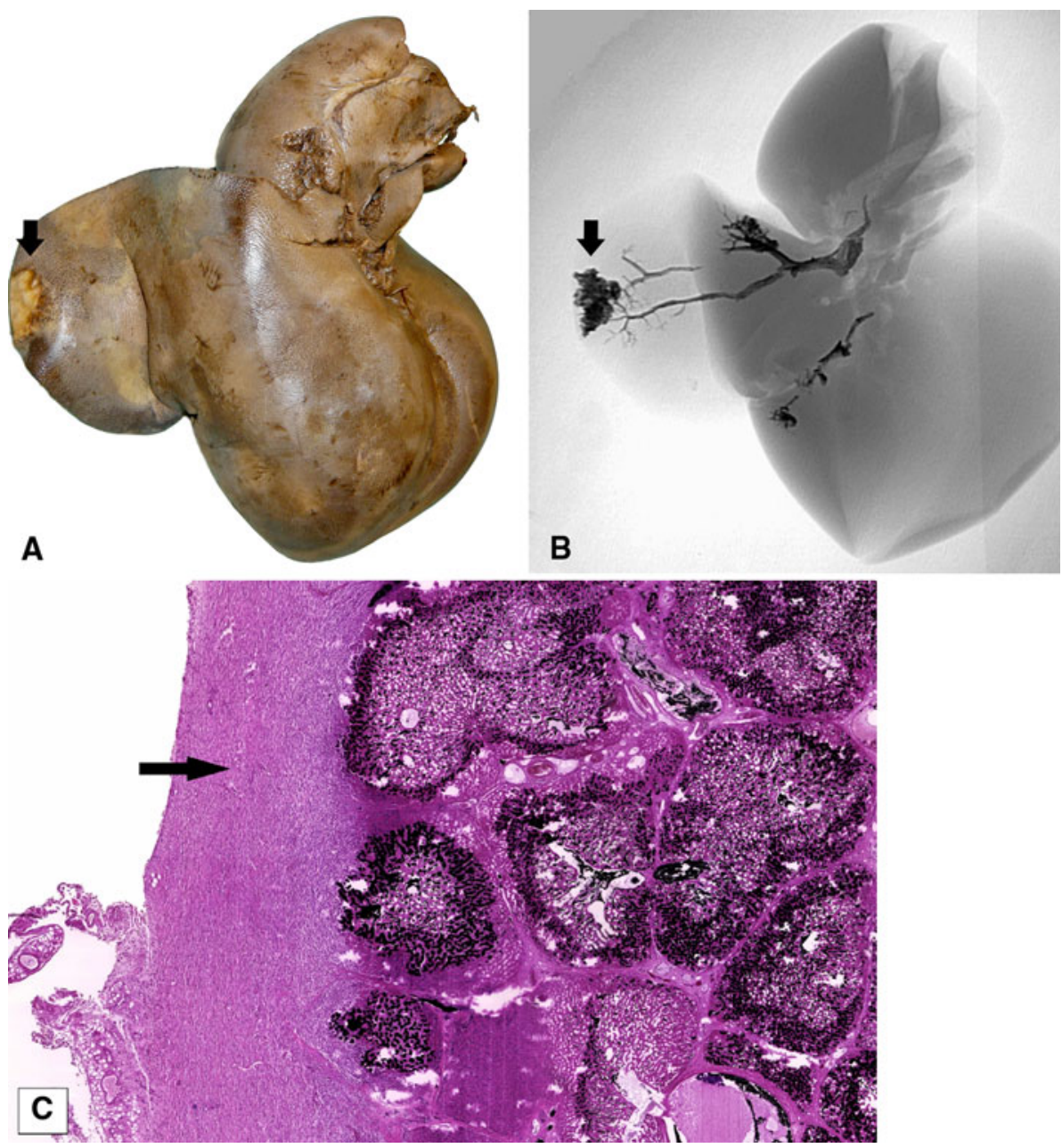

Histopathological assessment revealed that a mean number $(n)$ of 33 Onyx-filled lobules (range 20-49) were found per injection site. This is the number of lobules involved in the trajectory of Onyx toward the portal vein, as estimated with the fractal law formula. Some lobules were completely filled, i.e., Onyx in the central vein, the sinusoids, and the portal vein, whereas some lobules were only partially filled. At certain injection sites, the central veins of several lobules were dilated, reaching a tenfold size. Their surrounding sinusoids often were compressed and not filled with Onyx (Fig. 4). Distal to the injection site, Onyx was present only in the portal veins and not in the central veins, hepatic veins, or sinusoids. There was focal granulocytic accumulation around the Onyx-filled veins of the two pigs that were killed the day of the procedure. Granulation tissue and multinucleated giant cell reactions were visible in the wall of the central veins and portal veins of the embolized lobes of the pigs that were sacrificed at 7 and 21 days postprocedure. The embolized vessels showed no signs of recanalization. There were no signs of abscess formation in any of the livers and the nonembolized lobes were free of Onyx. The sinusoids in embolized and nonembolized segments were congested with blood, as seen in human livers after PVE.

\section{Discussion}

In the porcine model, we demonstrated that the portal vein can be embolized by retrograde transsinusoidal injection of Onyx. In $67 \%$ of the embolization attempts, Onyx was smoothly advanced into the portal venules and veins up to the origin of the segmental portal vein. In this way, the portal supply of at least one (37\%) and maximal two liver lobes $(63 \%)$ was blocked.

Onyx was considered a good candidate agent for transsinusoidal PVE, because it is a low-viscosity liquid embolic agent that has the ability to penetrate deep into complex vascular structures, such as cerebral arteriovenous malformations (AVM) [16, 20, 22]. Furthermore, the progression of this highly radiopaque agent can be accurately monitored under fluoroscopy. Another important feature of Onyx is its characteristic way of solidification. Inside the blood vessel, Onyx forms a coherent, spongy embolus that 


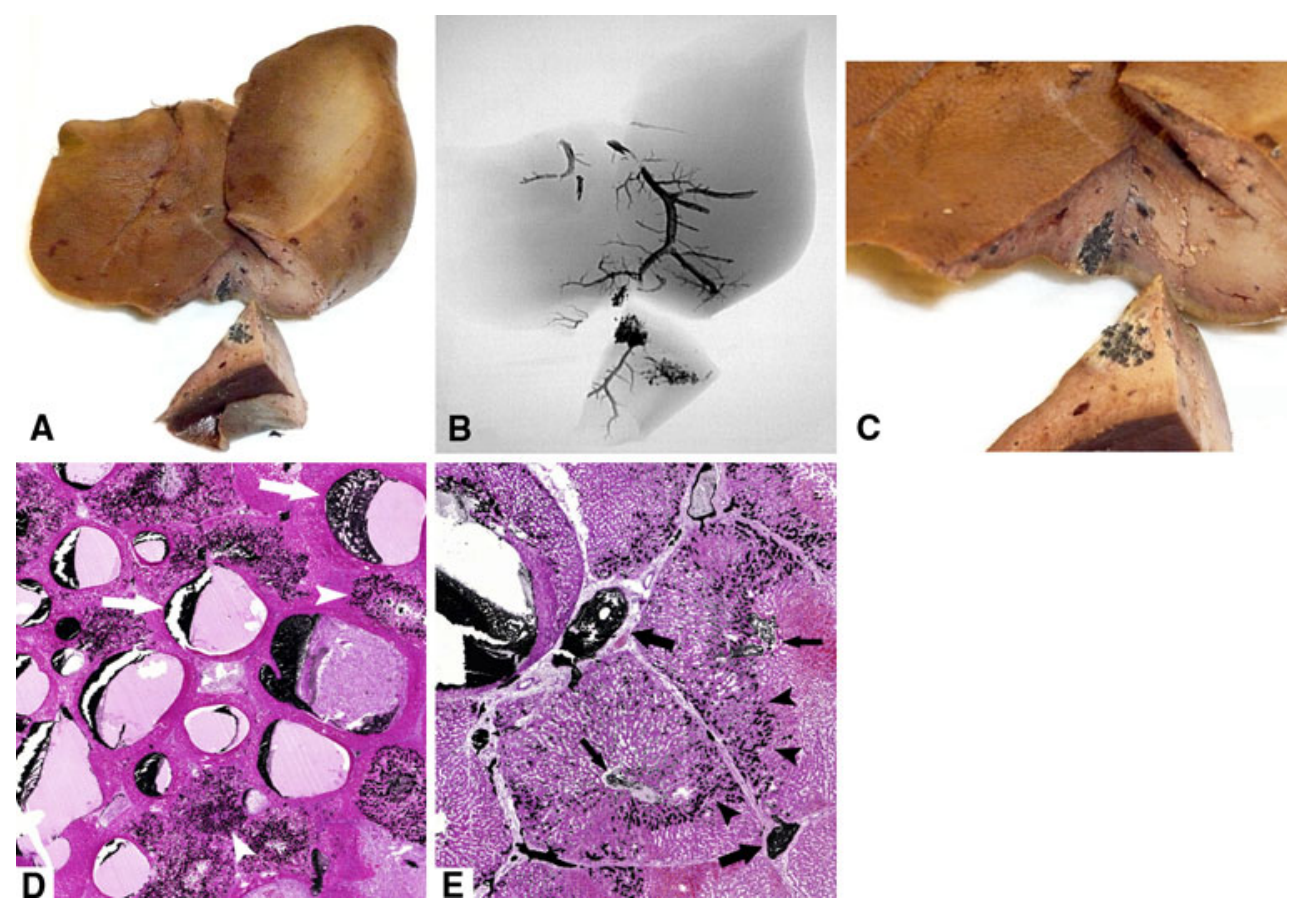

Fig. 4 A Macroscopic image of a resected liver with (B) the related radiographic image. C Close-up of injection area in (A). D Microscopic view $(H \& E$, original magnification $\times 40)$ near the injection site shows a combination of lobules with normal-sized central veins and Onyx-filled sinusoids (arrowheads) and lobules with dilated central veins and empty, suppressed sinusoids (arrows). E Lobules near the injection site $(\mathrm{H} \& \mathrm{E}$, original magnification $\times 40)$; Onyx is present in the central veins (thin arrows), in the sinusoids (arrowheads), and in the portal veins (thick arrows)

and error, guided by the progress of Onyx on fluoroscopy. Although Onyx was injected at a slow rate of approximately $0.1 \mathrm{ml} / \mathrm{min}$ in all procedures, there was variation in the interval between Onyx injection and its appearance in the portal system. At certain injections, Onyx moved through the sinusoids into the portal vein almost instantly after having filled only a small area of lobules. At other sites, a larger area of lobules had to be filled before Onyx reached the portal vein. A possible explanation for this observation is that the wedged microcatheter might not have fully occupied the vessel lumen and that Onyx leaked back up to the level of the wedged guiding catheter. Reflux was not always easily recognizable, because the tip of the microcatheter was near the tip of the guiding catheter. Furthermore, a large area of Onyx-filled lobules could obscure reflux on fluoroscopy. We experienced difficulties to differentiate filling of an adjacent hepatic vein through veno-venous communications from portal filling.

We estimated and histopathologically verified the number of lobules that are filled with Onyx around the injection site, because these lobules are completely embolized and will lose their function. The fractal law formula to estimate the number of lobules that are involved at each injection proved to be fairly accurate. The estimated number of involved lobules of 23 was in the range of the histopathologically counted number of Onyx-filled 
lobules (mean 33; range 20-59). This means that compared to the approximately one million lobules of which an average human liver consists [23], only a relatively small number of lobules lie distal to the tip of the microcatheter and a relatively small number of lobules are used (and sacrificed) to transport Onyx to the portal side. Reflux might be the reason for a larger number of Onyx-filled lobules at certain injection sites. In case of reflux, Onyx spreads to higher branches of the wedged hepatic vein and therefore more lobules are perfused. There might be a certain number of lobules critical for successful filling of the portal vein.

As the lobar portal veins in pigs are all separately connected to the portal trunk, we had to perform multiple injections to embolize more than one liver lobe. For instance, we could not advance Onyx from the right lateral lobe to the right medial lobe because the portal trunk would then be embolized as well. This would not be a problem on the left side where Onyx can be advanced from the left lateral lobe to the left medial lobe without embolizing the proximal part of the portal trunk that supplies the right lobes. In humans, the purpose of PVE often is to embolize the entire right or left lobe. Therefore, by advancing Onyx up to the right-left bifurcation of the portal vein, a single retrograde injection would theoretically be sufficient as long as there are no anatomical variations, such as a portal trifurcation. In our series, there was one case where the Onyx embolus extended into the portal trunk. The FLR relies on the blood supply from the portal trunk; therefore, it is of major importance that the portal trunk stays patent. This complication could possibly have been avoided by more carefully monitoring the extent of embolization or by using a DSA overlay of the indirect portography.

On histopathological examination, we observed a combination of empty lobules with dilated central veins and completely filled lobules near the injection site. We hypothesize that Onyx was not able to transverse the sinusoids of these empty lobules and, as a result, accumulated in the central veins. Then, Onyx probably followed the route of the least resistance and perfused other lobules that were more permeable to Onyx. Although Onyx was injected very gently during all procedures, the dilated central veins and the macroscopic scarring of the liver capsule present in two pigs indicate the impact of transsinusoidal PVE on parenchyma surrounding the injection site. Despite the fact that the central veins were approximately tenfold enlarged, the lobules were still intact. Furthermore, the macroscopic lesions of the liver capsule were firmly encased in fibrotic tissue without any signs of rupture of the liver capsule. Nevertheless, the damage to the liver and the risk of capsule rupture remains a point of attention.
Hypertrophy as an effect of transsinusoidal PVE with Onyx on the nonembolized part of the liver was not assessed, because this was not the purpose of this feasibility study. Next to complete occlusion of the portal supply, an inflammatory reaction of the perivascular tissue to the embolic agent might be an important factor for inducing hypertrophy. A moderate inflammatory reaction of the perivascular tissue and foreign body giant cell reactions were seen in the resected livers of the pigs sacrificed at 7 and 21 days postprocedural. These are known reactions to Onyx that can probably be ascribed to the DMSO-component $[16,18,24] . n \mathrm{BCA}$ is known to cause a strong inflammatory reaction as well [25], and in a comparative study of different embolic agents by de Baere et al. [26], livers embolized with $n \mathrm{BCA}$ exhibited the highest hypertrophy rate. The inflammatory reaction to $n \mathrm{BCA}$ is thought to induce the release of tumor necrosis factor (TNF) and interleukin-6, which stimulate hypertrophy of the nonembolized liver lobe. The acute inflammatory reaction noted after embolization with Onyx also might be a favorable factor for contralateral hypertrophy.

The results of this pig study cannot directly be translated to humans. One of the differences between the porcine liver and the human liver is the presence of a fibrous delineation around the lobules of the pig liver [27]. It is uncertain whether this fibrous layer influences the way Onyx can be advanced through the lobules. Another uncertainty is the level of anesthesia required during and after the procedure. From our experience with Onyx embolization in humans under local anesthesia, we know that DMSO can cause a painful reaction. Taking the focal swelling at the injection sites into account, we assume that transsinusoidal PVE may provoke capsular irritation of the liver. It is, therefore, questionable whether transsinusoidal PVE can be performed under local anesthesia. Further studies will have to prove the reproducibility of transsinusoidal PVE and its efficacy in inducing contralateral hypertrophy.

In conclusion, this study demonstrates transsinusoidal PVE to be a feasible procedure in pigs with a moderate success rate. This minimally invasive technique to embolize the portal vein can potentially be an alternative to the transhepatic approach. Complications of transsinusoidal PVE and the effect on contralateral hypertrophy warrant further study in animals.

Acknowledgments Ev3 (Irvine, CA, USA) is gratefully acknowledged for funding the equipment, materials, accommodation, and travel expenses involved in the research described in this article. The study of MS was supported by a research grant from the Dutch Cancer Society (Koningin Wilhelmina Fonds kankerbestrijding, Amsterdam, The Netherlands). 
Conflict of interest The authors declare that they have no conflict of interest.

Open Access This article is distributed under the terms of the Creative Commons Attribution Noncommercial License which permits any noncommercial use, distribution, and reproduction in any medium, provided the original author(s) and source are credited.

\section{References}

1. Mullin EJ, Metcalfe MS, Maddern GJ (2005) How much liver resection is too much? Am J Surg 190:87-97

2. Abu Hilal M, Lodge JP (2008) Pushing back the frontiers of resectability in liver cancer surgery. Eur J Surg Oncol 34: 272-280

3. Abulkhir A, Limongelli P, Healey AJ et al (2008) Preoperative portal vein embolization for major liver resection: a meta-analysis. Ann Surg 247:49-57

4. Pamecha V, Glantzounis G, Davies N et al (2009) Long-term survival and disease recurrence following portal vein embolisation prior to major hepatectomy for colorectal metastases. Ann Surg Oncol 16:1202-1207

5. Giraudo G, Greget M, Oussoultzoglou E et al (2008) Preoperative contralateral portal vein embolization before major hepatic resection is a safe and efficient procedure: a large single institution experience. Surgery 143:476-482

6. Madoff DC, Hicks ME, Vauthey JN et al (2002) Transhepatic portal vein embolization: anatomy, indications, and technical considerations. Radiographics 22:1063-1076

7. de Baere T, Denys A, Madoff DC (2007) Preoperative portal vein embolization: indications and technical considerations. Tech Vasc Interv Radiol 10:67-78

8. Stefano DRD, de Baere T, Denys A et al (2005) Preoperative percutaneous portal vein embolization: evaluation of adverse events in 188 patients. Radiology 234:625-630

9. Perarnau JM, Daradkeh S, Johann M et al (2003) Transjugular preoperative portal embolization (TJPE) a pilot study. Hepatogastroenterology 50:610-613

10. Madoff DC, Gupta S, Pillsbury EP et al (2007) Transarterial versus transhepatic portal vein embolization to induce selective hepatic hypertrophy: a comparative study in swine. J Vasc Interv Radiol 18:79-93

11. Krajina A, Lojik M, Chovanec V et al (2002) Wedged hepatic venography for targeting the portal vein during TIPS: comparison of carbon dioxide and iodinated contrast agents. Cardiovasc Intervent Radiol 25:171-175

12. Maleux G, Nevens F, Heye S et al (2006) The use of carbon dioxide wedged hepatic venography to identify the portal vein: comparison with direct catheter portography with iodinated contrast medium and analysis of predictive factors influencing level of opacification. J Vasc Interv Radiol 17:1771-1779

13. Debernardi-Venon W, Bandi JC, Garcia-Pagan JC et al (2000) $\mathrm{CO}(2)$ wedged hepatic venography in the evaluation of portal hypertension. Gut 46:856-860

14. Court FG, Wemyss-Holden SA, Morrison CP et al (2003) Segmental nature of the porcine liver and its potential as a model for experimental partial hepatectomy. Br J Surg 90:440-444

15. Camprodon R, Solsona J, Guerrero JA et al (1977) Intrahepatic vascular division in the pig: basis for partial hepatectomies. Arch Surg 112:38-40

16. Murayama Y, Vinuela F, Ulhoa A et al (1998) Nonadhesive liquid embolic agent for cerebral arteriovenous malformations: preliminary histopathological studies in swine rete mirabile. Neurosurgery 43:1164-1175

17. Ev3 (2011) Onyx ${ }^{\circledR}$ liquid embolic system, instructions for use. www.ev3.net. Accessed 28 April 2011

18. Komemushi A, Tanigawa N, Okuda Y et al (2002) A new liquid embolic material for liver tumors. Acta Radiol 43:186-191

19. Seldinger SI (1953) Catheter replacement of the needle in percutaneous arteriography: a new technique. Acta Radiol 39: 368-376

20. Katsaridis V, Papagiannaki C, Aimar E (2008) Curative embolization of cerebral arteriovenous malformations (AVMs) with Onyx in 101 patients. Neuroradiology 50:589-597

21. West GB, Brown JH, Enquist BJ (1997) A general model for the origin of allometric scaling laws in biology. Science 276:122-126

22. Wright KC, Greff RJ, Price RE (1999) Experimental evaluation of cellulose acetate NF and ethylene-vinyl alcohol copolymer for selective arterial embolization. J Vasc Interv Radiol 10: $1207-1218$

23. Krstic RV (1991) Digestive apparatus. In: Human microscopic anatomy: an atlas for students of medicine and biology. Springer, Berlin, p 224

24. Duffner F, Ritz R, Bomemann A et al (2002) Combined therapy of cerebral arteriovenous malformations: histological differences between a non-adhesive liquid embolic agent and $n$-butyl 2-cyanoacrylate (NBCA). Clin Neuropathol 21:13-17

25. Levrier O, Mekkaoui C, Rolland PH et al (2003) Efficacy and low vascular toxicity of embolization with radical versus anionic polymerization of $n$-butyl-2-cyanoacrylate (NBCA). An experimental study in the swine. J Neuroradiol 30:95-102

26. de Baere T, Denys A, Paradis V (2009) Comparison of four embolic materials for portal vein embolization: experimental study in pigs. Eur Radiol 19(6):1435-1442

27. Ekataksin W, Wake K (1991) Liver units in three dimensions: I. Organization of argyrophilic connective tissue skeleton in porcine liver with particular reference to the "compound hepatic lobule". Am J Anat 191:113-153 